\title{
Estudo das atipias indeterminadas em relação à prevalência e ao percentual de discordância nos casos do Programa de Prevenção do Câncer Uterino do Paraná
}

\section{Study of undetermined atypias in relation to prevalence and disagreement percentile in cases of the Cervical Cancer Screening Program of Paraná, Brazil}

Ana Paula Martins Sebastião'; Lúcia de Noronha²; Darliany Louise Hübner Scheffel3; Mariana Jorge Garcia ${ }^{3}$; Newton Sérgio de Carvalho ${ }^{4}$; Luiz Martins Collaço4; Luiz Fernando Bleggi-Torres ${ }^{5}$

\begin{tabular}{|c|c|}
\hline & \\
\hline $\begin{array}{l}\text { itologia cervicovaginal } \\
\text { revalência } \\
\text { ercentual de discordância } \\
\text { âncer do colo uterino } \\
\text { tipias de significado } \\
\text { indeterminado em células } \\
\text { escamosas (AsCUS) } \\
\text { Itipias de significado } \\
\text { indeterminado em células }\end{array}$ & $\begin{array}{l}\text { trodução: O Sistema Bethesda criou a categoria de atipias de significado indeterminado para albergar o } \\
\text { agnóstico citológico de alterações limítrofes entre o processo reacional e o neoplásico. Este estudo tem } \\
\text { mo objetivo avaliar a prevalência e o percentual de discordância dos casos de atipias indeterminadas em } \\
\text { lulas escamosas (ASCUS) e em células glandulares (AGUS) do Programa de Prevenção do Câncer de Colo } \\
\text { erino do Paraná (PPCUPR) que foram revistos pela Unidade de Monitoramento Externo da Qualidade } \\
\text { tológica (UMEQC). Da mesma forma tem como objetivo avaliar o padrão de reclassificação entre os casos } \\
\text { atipias indeterminadas (AI), que foram discordantes. Material e método: A UMEQC revisou } 65.753 \text { casos } \\
\text { Im período de } 25 \text { meses, dos quais foram selecionados casos de Al. Esses casos foram classificados em } \\
\text { ncordantes e discordantes para a obtenção da prevalência de cada um deles. Resultados: O total de Al } \\
\text { tes e após a revisão foi } 4.067 \text { casos (5,45\%) e } 3.584 \text { (6,19\%), respectivamente. O diagnóstico de ASCUS } \\
\text { rrespondeu, após a revisão, a 4,91\% (3.235) do total da amostra; e o de AGUS, a 0,51\% (338). Após a } \\
\text { classificação dos casos discordantes, de acordo com o padrão estabelecido pela UMEQC, o diagnóstico } \\
\text { gativo foi responsável pela maioria (57,32\%) daqueles que deixaram o grupo das Al. O diagnóstico } \\
\text { imário agrupado de neoplasia intra-epitelial cervical grau I (NIC I) e vírus do papiloma humano (HPV) } \\
\text { responsável pela maioria (51,1\%) dos casos que foram reclassificados como Al. Discussão e conclusões: } \\
\text { tre todos os casos discordantes as Al ocupam o sequndo luqar em freqüência. }\end{array}$ \\
\hline
\end{tabular}

abstract

Background: The Bethesda Pap Smear System introduced two categories of undetermined atypias: atypical squamous cells of undetermined significance (ASCUS) and atypical glandular cells of undetermined significance (AGUS). The objective of this study is to report the prevalence and the disagreement percentile of ASCUS and AGUS as diagnosed by the Cervical Cancer Screening Program of Paraná and revised by the Unit for External Monitoring of Cytology Quality (UEMCQ). Methods: The UEMCQ revised 65,753 cervical smears during 25 months and all undetermined atypias were selected. These cases were classified according to their agreement or not and then prevalence was obtained. Results: Before the UEMCQ review, the number of undetermined atypias was 4,067 (5.45\%); this number decreased to 3,584 after revising the data. Considering the whole sample, the ASCUS rate was $4.91 \%(3,235)$ and AGUS corresponded to only $0.51 \%$ (338). Most of the reclassified cases presented negative diagnoses (57.32\%). The primary grouped diagnoses, including CIN I and HPV, was responsible for $51.1 \%$ of undetermined atypias after reclassification. Discussion and conclusion: Undetermined atypias were the second most frequent among disagreement diagnosis groups.

key words

Cervical cytology

Prevalence

Disagreement percentile

Cervical cancer

Atypical squamous cells of

undetermined significance

(ASCUS)

Atypical glandular cells of

undetermined significance

(AGUS)

1. Mestra em Patologia.

2. Professora-adjunta da disciplina de Patologia da Pontifícia Universidade Católica do Paraná (PUC/PR) e da Universidade Federal do Paraná (UFPR).

3. Acadêmica de Iniciação Científica da PUC/PR.

4. Professor-adjunto da UFPR.

5. Professor-titular da disciplina de Patologia da PUC/PR e da UFPR.

Tese de mestrado apresentada na UFPR no ano de 2003. 


\section{Introdução}

A prevenção e o diagnóstico precoce constituem as formas ideais para reduzir a morbidade e a mortalidade decorrentes das neoplasias do colo uterino, sobretudo nos países em desenvolvimento. Programas de rastreamento do câncer do colo uterino têm significativa importância pelo fato de interromperem a história natural da doença, pois detectam neoplasias ou carcinoma in situ ainda em fase pré-invasora. Com tratamento adequado das lesões acima referidas, o câncer invasor pode ser evitado, reduzindo-se assim a mortalidade causada por essa doença(2).

O Sistema Bethesda incluiu, em 1988, um termo para uniformizar e normatizar o achado de alterações citológicas de caracterização indefinida ou limítrofes entre reativas e neoplásicas, denominando ASCUS (atypia in squamous cells of undetermined significance) as alterações de caracterização indefinida em células escamosas e AGUS (atypia in glandular cells of undetermined significance) as alterações achadas em células glandulares. As duas categorias, portanto, correspondem a achados citológicos indeterminados, para cuja definição etiológica se recomenda melhor investigação clínica(10).

A grande preocupação que a categoria atipias indeterminadas gera é a de ser usada como via de saída para as limitações de quem interpreta a citologia cervicovaginal, pois em reexames histológicos não é raro serem encontradas alterações menores diagnosticadas primariamente como ASCUS ou AGUS, erro freqüentemente marcado pela subjetividade do observador, o que pode resultar em supertratamento e estresse para as pacientes ${ }^{(17)}$.

Por essa razão, vários programas de prevenção do câncer de colo uterino em todo mundo começaram a utilizar programas de controle de qualidade para garantir a credibilidade dos exames citopatológicos, principalmente durante as campanhas intensivas ${ }^{(1,2,11,19)}$.

O Programa de Prevenção do Câncer do Colo Uterino do Paraná (PPCUPR) desenvolveu, desde o seu início, uma Unidade de Monitoramento Externo de Qualidade Citológica (UMEQC), a fim de controlar a qualidade dos diagnósticos emitidos. A UMEQC concentra suas atividades no Hospital de Clínicas da Universidade Federal do Paraná (HC-UFPR) através de um termo de cooperação com a Secretaria de Saúde do Paraná. Na excelência e na contínua atualização dos médicos e professores ligados à Seção de Citopatologia do Serviço de Anatomia Patológica do HC-UFPR se sustenta o reconhecimento de notório saber a esse serviço, necessário para pôr em prática um sistema de fiscalização de qualidade. Essa equipe é constituída por dois revisores especiais - patologistas com formação específica em citopatologia, membros da Sociedade Brasileira de Citopatologia e Members of International Academy of Citology (MIAC) - que exercem a coordenação, e por sete revisores gerais - patologistas gerais com formação em citopatologia.

O banco de dados da UMEQC contava com 65.753 casos quando tais casos foram compilados para a elaboração deste trabalho. Foram escolhidos os casos de ASCUS e AGUS para uma análise mais aprofundada, devido às controvérsias no que se refere à aplicação dos critérios morfológicos para o diagnóstico e seu significado clínico.

O objetivo deste estudo é avaliar a prevalência e o percentual de discordância dos casos de atipias indeterminadas escamosas e glandulares do Programa de Prevenção do Câncer de Colo Uterino do Paraná (PPCUPR), antes e após a revisão pela Unidade de Monitoramento Externo da Qualidade Citológica (UMEQC), e compará-los com os dos demais grupos diagnósticos, e, ainda, estabelecer entre os casos discordantes de atipias indeterminadas o padrão de reclassificação.

\section{Material e método}

A UMEQC tem como finalidade principal detectar falsos negativos e falsos positivos, que poderiam acarretar tratamentos clínicos inadequados. Para isso ela se propõe revisar cerca de $10 \%$ dos laudos citopatológicos emitidos pelo PPCUPR, a cada mês, utilizando dois métodos de busca de tais exames: busca ativa (todos os exames com qualquer tipo de alteração celular devem ser revistos, inclusive os classificados nos grupos 2, 3, 4 e 5) (Tabela 1) e escolha aleatória, pela qual aos de busca ativa soma-se um número variável de exames citológicos do grupo 1 (Tabela 1), selecionados de forma aleatória, até o total de $10 \%$ dos exames mensais de cada patologista).

Foram selecionados, a partir de um banco de dados da UMEQC/PPCUPR composto por 65.753 casos, todos os casos de citologia oncótica cervicovaginal com resultado de atipias indeterminadas, os quais foram sistematicamente submetidos à avaliação pela UMEQC por um período consecutivo de dois anos (setembro de 1997 a outubro de 1999). Os dados foram obtidos através da análise dos laudos dos diagnósticos primários e posteriormente dos laudos de revisão pela UMEQC.

Para o diagnóstico de ASCUS, AGUS e simultaneamente ASCUS e AGUS (ASCUS/AGUS) foram adotados os critérios 
citopatológicos descritos por Kurman e Solomon (1997) em publicação oficial do Sistema Bethesda ${ }^{(10)}$.

Os diagnósticos de revisão representam os diagnósticos finais emitidos pela UMEQC após a revisão das lâminas, e os diagnósticos primários são aqueles emitidos pelos patologistas do PPCUPR antes da revisão feita pela UMEQC. Ambos foram divididos em cinco categorias (ou grupos diagnósticos), com base no tipo de conduta clínica adotada pelo PPCUPR, sendo também subdivididos em diagnósticos negativos e não-negativos, conforme a Tabela 1.

Considerou-se que houve discordância diagnóstica quando o diagnóstico primário de atipias indeterminadas foi diferente do diagnóstico de revisão.

Chegou-se ao percentual de discordância na comparação entre as avaliações de grupos de diagnósticos primários em relação à proporção de discordância em cada grupo de diagnóstico de revisão.

A mudança de um diagnóstico primário para outra categoria diagnóstica de revisão é o que chamamos de realocação. Ela inclui as categorias: realocação positiva (os casos primários que migraram de outros grupos e foram acrescentados ao grupo 2 após a revisão) e realocação negativa (que são os casos com diagnóstico primário de grupo 2 que migraram para outros grupos depois da revisão).

As variáveis do estudo, por serem de natureza categórica, foram expressas em freqüências e percentuais. Para as comparações entre os grupos diagnósticos e categorias diagnósticas considerou-se a odds ratio estimada através de um modelo de regressão logística. Para identificação da homogeneidade das distribuições de categorias ou grupos diagnósticos adotou-se o teste de qui-quadrado. A proporção de casos de ASCUS e de AGUS concordantes em amostras satisfatórias, assim como a de casos realocados, foi expressa por meio de intervalos de $95 \%$ de confiança.
Quando o intervalo de confiança não é o de 0,5 (ou 50\%) fica indicado que a proporção entre as amostras avaliadas é significativamente diferente entre elas. Em todos os testes um valor de $p<0,05$ foi considerado estatisticamente significante.

\section{Resultados}

\section{Avaliação da prevalência dos casos de atipias indeterminadas escamosas e glandulares do PPCUPR antes e depois da revisão pela UMEQC e comparação com os demais grupos diagnósticos}

A quantidade de casos em cada grupo diagnóstico antes e depois da revisão pela UMEQC está representada na Tabela 2. Observa-se que há variação da quantidade de exames em cada grupo diagnóstico, para mais e para menos, como resultado das realocações positivas e negativas, respectivamente. Nos Grupos 1 e 4, a quantidade de diagnósticos após a revisão foi maior, ou seja, houve mais realocações positivas que negativas; nos demais grupos, após a revisão houve diminuição na quantidade de casos, indicando que houve mais realocações negativas. Focando especificamente o Grupo 2, verifica-se que a diferença percentual entre o diagnóstico primário e o diagnóstico de revisão é de $1,2 \%$ (NS- $p=0,0504)$. Esse percentual indica que o número das realocações negativas foi maior que $\mathrm{o}$ das positivas.

Os diagnósticos primários de ASCUS correspondem a $5,68 \%$ (3.736 casos) do total de 65.753 estudados, e os de AGUS, a 0,48\% (316 casos). Após a revisão pela UMEQC, o número total de ASCUS foi de 3.235, 4,91\% do total da amostra; o número de AGUS aumentou para 338 casos, correspondendo a $0,51 \%$ do total da amostra.

\section{Tabela 1 Grupos diagnósticos}

Diagnóstico negativo

Grupo 1: Negativo para malignidade. Inclui ausência de células anormais ou alterações reativas ou reacionais

Diagnóstico não-negativo

Grupo 2: Alterações limítrofes. Incluem atipias indeterminadas escamosas (ASCUS) e glandulares (AGUS)

Grupo 3: Lesões de baixo grau. Incluem alterações citopáticas compatíveis com vírus do papiloma humano (HPV) e neoplasia intra-epitelial cervical grau I (NIC I) Grupo 4: Lesões de alto grau e carcinomas. Incluem neoplasia intra-epitelial cervical graus II (NIC II) e III (NIC III), carcinoma escamocelular invasor (CEC) e adenocarcinoma

Ausência de diagnóstico Grupo 5: Esfregaços insatisfatórios 
Na Tabela 3, observa-se a distribuição dos diagnósticos de ASCUS e AGUS antes e depois da revisão pela UMEQC.

Após a revisão pela UMEQC, houve aumento do percentual de diagnósticos AGUS. Tal achado indica que foram feitas mais realocações negativas nos diagnósticos de ASCUS e mais realocações positivas nos de AGUS.

\begin{tabular}{|c|c|c|c|c|}
\hline \multirow{3}{*}{$\begin{array}{l}\text { Tabela } 2 \\
\text { Grupo } \\
\text { diagnóstico }\end{array}$} & \multicolumn{4}{|c|}{$\begin{array}{l}\text { Distribuição dos diagnósticos } \\
\text { citológicos agrupados de todos os } \\
\text { casos }(n=65.753 \text { ) antes e depois } \\
\text { da revisão feita pela UMEQC, } \\
\text { número absoluto e porcentagem } \\
\text { em relação ao total em cada grupo } \\
\text { (coluna) }\end{array}$} \\
\hline & \multicolumn{2}{|c|}{$\begin{array}{c}\text { Diagnóstico } \\
\text { primário }\end{array}$} & \multicolumn{2}{|c|}{$\begin{array}{l}\text { Diagnóstico } \\
\text { de revisão }\end{array}$} \\
\hline & $n$ & $\%$ & $n$ & $\%$ \\
\hline Grupo 1 & 54.959 & 83,58 & 55.583 & 84,53 \\
\hline Grupo 2 & 4.067 & 6,19 & 3.584 & 5,45 \\
\hline Grupo 3 & 3.008 & 4,57 & 2.523 & 3,84 \\
\hline Grupo 4 & 2.526 & 3,84 & 2.892 & 4,4 \\
\hline Grupo 5 & 1.193 & 1,81 & 1.170 & 1,78 \\
\hline Total & 65.753 & 100 & 65.752 & 100 \\
\hline
\end{tabular}

Fonte: UMEQC.

Tabela 3

Distribuição dos casos de atipias indeterminadas antes $(n=4.067)$ e depois $(n=3.584)$ da revisão feita pela UMEQC, por entidade

\begin{tabular}{lccccc}
\hline \multirow{2}{*}{$\begin{array}{l}\text { Entidade } \\
\text { diagnóstica }\end{array}$} & \multicolumn{2}{c}{$\begin{array}{c}\text { Diagnóstico } \\
\text { primário }\end{array}$} & & \multicolumn{2}{c}{$\begin{array}{c}\text { Diagnóstico } \\
\text { de revisão }\end{array}$} \\
\cline { 2 - 3 } \cline { 5 - 6 } & $n$ & $\%$ & & $n$ & $\%$ \\
ASCUS & 3.736 & $91,86^{*}$ & & 3.235 & $90,26^{*}$ \\
AGUS & 316 & $7,76^{* *}$ & & 338 & $9,43^{* *}$ \\
ASCUS/AGUS*** & 15 & 4,57 & & 11 & 3,84 \\
$\begin{array}{l}\text { Total de atipias } \\
\text { indeterminadas }\end{array}$ & 4.067 & 100 & & 3.584 & 100 \\
\hline
\end{tabular}

Fonte: UMEQC; ${ }^{*} \mathrm{p}=0,0142 ;{ }^{* *} \mathrm{p}=0,0091 ;{ }^{* * *}$ diagnóstico simultâneo de ASCUS e AGUS.

\section{Avaliação do percentual de discordância entre os diagnósticos antes e depois da revisão pela UMEQC e comparação com os demais grupos diagnósticos}

Observando a Tabela 4, que demonstra o percentual de discordância para cada grupo diagnóstico, pode-se observar que o Grupo 2 foi o segundo mais discordante $(p<0,001)$ quando pareados os grupos dois a dois.

Na Tabela 5, quando comparados os casos de ASCUS, AGUS e ASCUS/AGUS com as demais entidades diagnósticas, o valor de $p$ foi estatisticamente significativo quando se comparou o grupo ASCUS com HPV, NIC II e NIC III. No entanto, com relação ao AGUS, demonstrou-se significância estatística entre essa entidade e HPV, NIC I e adenocarcinoma. Todas as comparações com diagnósticos simultâneos de ASCUS/AGUS não mostraram significância estatística, exceto com os insatisfatórios, devido ao pequeno número de casos.

\section{Avaliação do padrão de reclassificação de casos discordantes envolvendo atipias indeterminadas}

As Tabelas 6 e 7 demonstram como foram redistribuídos os casos que sofreram realocação, tanto negativa quanto positiva, após a revisão pela UMEQC.

Por exemplo, 458 (57,32\%) casos do Grupo 2 sofreram realocação negativa para o Grupo 1 após a revisão pela UMEQC, sendo este o diagnóstico de revisão (classificação UMEQC - correta). Evidencia-se então que, havendo um caso do Grupo 2 com possibilidade de estar errado, a probabilidade maior é que ele seja realocado para o Grupo 1 (Tabela 6). Tabela 4 um dos grupos diagnósticos

Percentual de discordância entre 0 diagnóstico primário e 0 diagnóstico da UMEQC para cada

\begin{tabular}{lc}
\hline Grupo diagnóstico & Discordância (\%) \\
\hline Grupo 1 & 0,24 \\
Grupo 2 & 19,65 \\
Grupo 3 & 23,74 \\
Grupo 4 & 7,28 \\
Grupo 5 & 2,51 \\
\hline
\end{tabular}

Fonte: UMEQC; $\mathrm{p}<0,001$ 
Percentual de discordância para cada uma das entidades

Tabela 5 diagnósticas

\begin{tabular}{lcc}
\hline $\begin{array}{l}\text { Grupo } \\
\text { diagnóstico }\end{array}$ & $\begin{array}{c}\text { Entidade } \\
\text { diagnóstica }\end{array}$ & $\begin{array}{c}\text { Discordância } \\
(\%)\end{array}$ \\
\hline Grupo 1 & Negativo & 0,24 \\
\hline Grupo 2 & ASCUS & 21,2 \\
& AGUS & 11,08 \\
& ASCUS/AGUS* & 26,67 \\
\hline Grupo 3 & HPV & 41,04 \\
& NIC I & 19,42 \\
\hline Grupo 4 & NIC II & 12,7 \\
& NIC III & 7,56 \\
& CEC & 15,48 \\
& Adenocarcinoma* * & 45,46 \\
\hline Grupo 5 & Insatisfatório & 2,51 \\
\hline
\end{tabular}

Fonte: UMEQC; ASCUS: alterações celulares limítrofes em células escamosas; ACUS: alterações celulares limitrofes em células glandulares; HPV: vírus do papiloma humano; NIC: neoplasia intra-epitelial cervical; CEC: carcinoma escamocelular invasor; ASCUS/AGUS: diagnóstico simultâneo de ASCUS e AGUS; "número absoluto de casos antes da revisão $=15$, após a revisão $=11$; * número absoluto de casos antes da revisão $=22$, após a revisão $=14$.

\section{Tabela 6 segundo a UMEQC}

\section{Redistribuição dos diagnósticos} primários de atipias

indeterminadas (Grupo 2) quanto à realocação negativa

\begin{tabular}{lcc}
\hline Classificação UMEQC & \multicolumn{2}{c}{ Casos do Grupo 2 com } \\
Diagnóstico de revisão & $n$ & $\%$ \\
\hline Grupo 1 & $458^{*}$ & $57,32^{*}$ \\
Grupo 3 & 141 & 17,65 \\
Grupo 4 & 199 & $24,91^{*}$ \\
Grupo 5 & 1 & 0,13 \\
Total & $\mathbf{7 9 9}$ & \\
\hline
\end{tabular}

Fonte: UMEQC; ${ }^{*} \mathrm{p}<0,001$

Na Tabela 7, observa-se que é provável que um diagnóstico errado dos Grupos 1, 3, 4 e 5 seja realocado para - Grupo 2. As probabilidades dessas realocações positivas foram numérica e estatisticamente diferentes e significativas, exceto no pareamento entre o Grupo 1 e o Grupo 4, em que a probabilidade é igual para ambos e não é significativa. Em vista disso, podemos observar que, por exemplo,
Redistribuição dos diagnósticos primários de outros grupos diagnósticos responsáveis pela Tabela 7 realocação positiva para 0 Grupo 2

\begin{tabular}{lcc}
\hline Classificação inicial & $\begin{array}{c}\text { Casos do Grupo } 2 \text { com } \\
\text { realocação positiva }\end{array}$ \\
Diagnóstico primário & $n$ & $\%$ \\
Grupo 1 & $70(p=\mathrm{NS})^{*}$ & 21,8 \\
Grup0 3 & $161^{* *}$ & $51,1^{*}$ \\
Grup0 4 & $81(p=\mathrm{NS})^{*}$ & 25,6 \\
Grupo 5 & 5 & 1,5 \\
Total & 316 & 100 \\
\hline
\end{tabular}

Fonte: UMEQC; ${ }^{*} \mathrm{p}$ não estatisticamente significante; ${ }^{* *} \mathrm{p}<0,001$.

161 (51,1\%) casos do Grupo 2, que sofreram realocação positiva eram primariamente classificados como Grupo 3 (diagnóstico primário).

A prevalência real do diagnóstico de atipias indeterminadas sofreu influência tanto da realocação positiva quanto da exclusão de casos de realocação negativa.

\section{Discussão}

A importância do estudo de casos de atipias indeterminadas escamosas e glandulares do PPCUPR fica demonstrada pelo expressivo número de casos registrados no banco de dados da UMEQC e analisados neste trabalho, onde essa categoria (Grupo 2) ocupa o segundo lugar em freqüência diagnóstica (6,19\%). Ressalta-se a expressividade desse número, se considerarmos que ele só é menor que o número de casos negativos (Grupo 1), os quais representam a grande maioria dos exames citológicos cervicovaginais.

Após a revisão feita pela UMEQC, o Grupo 2 sofreu um decréscimo de freqüência de 1,2\%, significando que haviam sido feitos mais diagnósticos de atipias indeterminadas do que o considerado correto. Ainda assim, o percentual de atipias indeterminadas continua de acordo com o recomendado pelo Sistema Bethesda, segundo o qual essa categoria não deve representar mais do que $5 \%$ a $6 \%$ do número total de casos de um laboratório, ou duas a três vezes o número de casos de lesões intra-epiteliais escamosas de baixo grau diagnosticadas em um ano em determinado serviço.

O decréscimo de 1,2 ponto percentual na correção dos casos de atipias indeterminadas após a revisão pela UMEQC pode dar a falsa impressão de que elas não teriam sido adequadamente corrigidas. No entanto, essa porcentagem 
representou uma diferença de 19\% entre o diagnóstico primário e o revisado. Os casos de atipias indeterminadas ocuparam a segunda posição em discordância, ficando a diferença em menos de 5\% das lesões de baixo grau (Grupo $3)$, as mais freqüentes em discordância (23,74\%). Sendo assim, só o estudo mais detalhado desse grupo poderá revelar a importância dele na epidemiologia do câncer de colo, visto que as atipias indeterminadas ainda são uma entidade controversa e com várias dificuldades diagnósticas morfológicas.

Analisando cada entidade diagnóstica separadamente quanto ao percentual de discordância, deve-se considerar que altos percentuais de discordância foram justificados por pequeno número de casos envolvendo o diagnóstico de duas delas: o adenocarcinoma e as ASCUS/AGUS. É provável que a ocorrência de tais diagnósticos errôneos se deva à pouca experiência dos citopatologistas em diagnosticar lesões glandulares, já que elas são de ocorrência mais rara que as escamosas.

O diagnóstico de ASCUS (21,2\% de discordância), responsável pelo maior percentual de discordância depois do ASCUS/AGUS, tanto pode representar diversas lesões como também simples alterações reativas ou reparativas. Montz et al.(1992) encontraram 19\% de lesões intra-epiteliais escamosas em 91 casos em avaliação cito-histológica, enquanto Dvorak et al.(1999) obtiveram 18\% de casos de NIC em amostra de 249 pacientes e Collaço et al.(2000) encontraram $45 \%$ de NIC, $51 \%$ de cervicite acentuada e metaplasia escamosa e $4 \%$ de carcinoma invasor. Corroborando este último estudo, Lousuebsakul et al.(2000) encontraram $47 \%$ de lesões intra-epiteliais e $34 \%$ de alterações reativas em 421 casos, reforçando a diversidade de formas de lesões que alberga a categoria de alterações de significado indeterminado(12).

Esses dados justificam a proposta de Sherman et al.(1999) da subdivisão das ASCUS em ASCUS sem outra especificação e ASCUS com tendência para lesão intra-epitelial. Para sua classificação, a literatura valoriza achados comparativos citopatológicos e histológicos em fragmentos teciduais, metaplasia imatura com atipias, metaplasia madura com atipias, células pequenas atípicas e reparos com atipias, irregularidade da membrana nuclear $(7,16)$. Entretanto, em estudo anteriormente desenvolvido em que se avaliou a variabilidade interobservadores em 122 biópsias cervicais, concluiu-se que o grau de concordância é baixo nas diversas categorias, principalmente no grupo das atipias de significado indeterminado e no grupo dos negativos, demonstrando que a reprodutividade de tais diagnósticos é pobre, mesmo quando se utiliza a histopatologia ${ }^{(4)}$.

Observa-se que a UMEQC realocou em outras categorias 799 casos de atipias indeterminadas, sendo que na maioria $(57,32 \%)$ eles foram reclassificados como negativos (Grupo 1), o que evitou custos desnecessários ao programa na busca ativa pelas pacientes, na repetição de exames e no seguimento. A preocupação por utilizar controle de qualidade diagnóstica visando a redução dos custos e da exposição das pacientes a tratamentos desnecessários já havia sido demonstrada por Seagnan et al.(1994), quando descreveram um programa de rastreamento de câncer de colo uterino na comunidade européia, para a avaliação dos custos e dos benefícios. Concluíram que para o êxito do programa é necessário o desenvolvimento de protocolos que considerem não só a efetividade do sistema, mas também os efeitos adversos que este pode causar. Tais programas implicam na adoção de mecanismos de controle de qualidade, acurácia na leitura do exame citológico e protocolos de acompanhamento. Outros autores obtiveram dados que corroboram os achados desta pesquisa, pois encontraram 12,5\% de exames superdiagnosticados quando analisaram ASCUS e lesões de baixo grau atribuindo a todos algum erro de interpretação(5). As alterações inflamatórias e reparativas, compreendendo o aumento nuclear, nucléolos proeminentes, lâminas celulares coesas com núcleos vesiculosos e por vezes pleomórficos, foram os critérios que mais freqüentemente levaram os patologistas a incluírem tais alterações na categoria de ASCUS ${ }^{(21)}$. Isso se deve à falta de dados clínicos precisos e ao pouco conhecimento das variantes qualitativas e quantitativas que as alterações reacionais e inflamatórias podem provocar nos esfregaços cervicovaginais. Freqüentemente os padrões histológicos são supervalorizados pelo receio de subestimar a severidade da lesão avaliada(3).

Neste estudo, a realocação de 141 casos (17,65\%) para o Grupo 3 e 199 casos (24,91\%) para o Grupo 4 demonstra a fundamental importância do estabelecimento de programas de controle de qualidade diagnóstica para atipias indeterminadas, pois as pacientes foram encaminhadas para tratamento específico, colposcopia e biópsia dirigida, ao invés de serem encaminhadas para seguimento ambulatorial com citologias.

Entre os diagnósticos que a UMEQC reclassificou como atipias indeterminadas (realocação positiva), a maioria $(51,1 \%)$ tinha sido diagnosticada como lesões de baixo grau (Grupo 3).

Diante de dúvida diagnóstica, enfatiza-se a necessidade de consultoria interpatologistas como tentativa de maior 
asseguramento quanto ao controle da qualidade do diagnóstico e à diminuição da taxa de erros ${ }^{(14,20)}$.

A diversidade na formação e na experiência dos patologistas gerais das várias cidades do Paraná que trabalham no PPCUPR reflete a realidade também encontrada no setor privado de laboratórios. Não há um método específico para avaliar o motivo das realocações negativas que determinaram a diminuição dos casos de atipias indeterminadas após a revisão pela UMEQC. Fatores como formação profissional, experiência, sobrecarga de trabalho, dificuldade diagnóstica e variação diagnóstica interobservadores interferem na avaliação dos casos considerados como atipias indeterminadas, deturpando a real importância e a função do emprego dessa classificação diagnóstica.

A variabilidade interobservadores é relatada em vários estudos de patologia e citopatologia pelo fato de se tratar de exames sujeitos a julgamento subjetivo e a uma pura interpretação e aplicação dos critérios diagnósticos descritos para cada entidade diagnóstico. Gatsha et al. (2001) realizaram estudo envolvendo 632 esfregaços com diagnóstico anterior de ASCUS, os quais foram revistos por outros três observadores. Entre os 200 casos (32\%) que apresentavam concordância completa interobservadores, 91 (45\%) diagnósticos primários de ASCUS foram confirmados, demonstrando a baixa reprodutividade dessa categoria diagnóstica ${ }^{(8)}$. Segundo estudos desenvolvidos por Smith et al. (2000), Juskevicius et al. (2001) e Stoler et al. (2001), as taxas de ASCUS e de lesão intra-epitelial escamosa são influenciadas pela rigidez na adoção de critérios morfológicos e pelo grau de experiência na interpretação dos espécimes citológicos, sendo muitas vezes desconhecidos os fatores que afetam a reprodutividade interobservadores ${ }^{(6,9,18)}$.

Finalizando, acredita-se que as taxas de diagnósticos de atipias indeterminadas são passíveis de redução quando há estreita relação entre o ginecologista e o patologista, cada um contribuindo dentro da sua especialidade. Ao ginecologista cabe fornecer informações clínicas relevantes e realizar coleta abrangente e de boa qualidade, observando os fatores que possam limitar a qualidade de uma amostra cervicovaginal. Ao patologista cabe a busca pelo aprimoramento dos seus critérios diagnósticos, cautela nos casos com alterações limítrofes e compartilhamento de dúvidas com os colegas da área. As observações feitas neste estudo identificam também os fatores que merecem maior atenção por parte dos profissionais envolvidos com o citodiagnóstico.

Assim, o bom relacionamento e o entrosamento dessas duas especialidades podem contribuir muito para a melhora do diagnóstico e, conseqüentemente, do manejo de pacientes portadoras de atipias indeterminadas.

\section{Conclusões}

A prevalência de atipias indeterminadas em citologia cervicovaginal (Grupo 2) foi de 3.584 casos, ou seja, 5,45\% do total da amostra de 65.753 casos. Desses 3.584 casos de atipias indeterminadas, 90,26\% foram representados por diagnósticos de ASCUS, 9,43\% por diagnósticos de AGUS e $3,84 \%$ de ASCUS/AGUS.

Houve diminuição de 1,2\% ( $p=\mathrm{NS}$ ) na quantidade de casos de atipias indeterminadas (Grupo 2) após a revisão pela UMEQC, sendo que houve aumento do percentual de diagnósticos de AGUS e diminuição do percentual de diagnósticos de ASCUS.

O percentual de discordância para o grupo de atipias indeterminadas (Grupo 2) foi de 19,65\%, sendo que o padrão de realocação mostrou que o diagnóstico negativo (Grupo 1) foi responsável pela maioria das realocações negativas $(57,32 \%)$ e o diagnóstico primário agrupado de NIC I e HPV (Grupo 3) foi responsável pela maioria das realocações positivas $(51,1 \%)$.

\section{Referências}

I. ALVES, V. A. F. et al. Programa de controle de qualidade ginecológica do Instituto Adolfo Lutz: estratégias e análise crítica dos resultados de sua implantação-piloto. Revista da Associação Médica do Brasil, v. 37, n. I, I991.

2. ANDERSON, G. H. Cytologic screening programs. In: BIBBO, M. Comprehensive cytopathology. Philadelphia: WB Saunders, 1991. p. 48-53.

3. COLLAÇO, L. M. et al. Correlação cito-histológica em 326 pacientes submetidas a cirurgia de alta freqüência (CAF) no Programa de Câncer Ginecológico do Estado do Paraná. J Bras Patol, v. 36, n. 3, 191-7, 2000.

4. CREAGH, T. et al. Pathologist variation in reporting cervical borderline epithelial abnormalities and cervical intraepithelial neoplasia. / Clin Pathol, v. 48, p. 59-60, 1995.

5. DUGGAN, M.A.; BRAASHER, P.M.Accuracy of Pap tests reported as CIN I. Diagn Cytopathol, v. 21, n. 2, p. 129-36, 1999.

6. DVORAK, K. A.; FINNEMORE, M.; MAKSEM, J. A. Histology correlation with atypical squamous cells of undetermined 
significance (ASCUS) and low-grade squamous intraepithelial lesion (LSIL) cytology diagnoses: an argument to ensure ASCUS follow-up that is as aggressive as that for LSIL. Diagn Cytopathol, v. 2 I, n. 4, p. 292-5, 1999.

7. ETTLER, H. C. et al. Atypical squamous cells of undetermined significance: a cytopatological study in a colposcopy clinic. Diagn Cytopathol, v. 21, n. 3, p. 21 I-6, 1999.

8. GATSHA, R. M. et al. Smears diagnosed as ASCUS: interobserver variation and follow-up. Diagn Cytopathol, v. 25, n. 2, p. 138 40, 2001 .

9. JUSKEVICIUS, R. et al. An analysis of factors that influence the ASCUS/SIL ratio of pathologists. Am J Clin Pathol, v. I 6, n. 3, p. 33 I-5, 200I.

10. KURMAN, R. J.; SOLOMON, D. O. Sistema Bethesda para o relato de diagnóstico citológico cervicovaginal. Rio de Janeiro: Revinter, 1997.

I I. LAZCANO-PONCE, E. C. et al.Validity and reproducibility of cytologic diagnosis in a sample of cervical cancer screening centers in Mexico. Acta Cytologica, v. 4I, n. 2, p. 277-84, 1997.

12. LOUSUEBSAKUL, V. et al. Clinical impact of atypical squamous cells of undetermined significance: a cytohistologic comparision. Acta Cytologica, v. 44, n. I, 2000.

13. MONTZ, F. J. et al. Natural history of the minimally abnormal Papanicolaou smear. Obstetrics \& Gynecology, v. 80, n. 3, 1992.
14. RENSHAW,A.A.Analysis of error in calculating the false-negative rate in the interpretation of cervicovaginal smears: the need to review abnormal cases. Cancer, v. 8I, n. 5, 264-7I, 1997.

15. SEAGNAN, N. Cervical cancer screening. Human benefits and human costs in the evaluation of screening programmes. Euro J Cancer, v. 30A, n. 6, p. 873-5, 1994.

16.SHERMAN, M. E. et al. "ASCUS, rule out HSIL": cytologic features, histologic correlates, and human papillomavirus detection. Mod Pathol, v. 12, n. 4, p. 335-42, 1999.

17. SMITH,A. E. et al. Review of the Bethesda System atlas does not improve reproducibility or accuracy in the classification of atypical squamous cells of undetermined significance smears. Cancer, v. 90, n. 4, p. 201-6, 2000.

18. STOLER, M. H.; SCHIFMAN, M. Atypical squamous cells of undetermined significance-low-grade squamous intraepithelial lesion triage study (ALTS) group: interobserver reproducibility of cervical cytology and histology interpretations; realistic estimates from the ASUS-LSIL Triage Study.JAMA, v. 285, n. I I, p. 1500-5, 200 I.

19.TRAVERS, H. Quality assurance indicators in anatomic pathology. Arch Pathol Lab Med, v. I |4, p. I |49-56, 1990.

20. VOOIJS, G. P. et al. A workstation for screening, supervision, reviewing, quality assurance and education in cytopathology. Acta Cytologica, v. 40, n. I, p. 9, 1996.

2I. WIED, G. L. et al. Compendium on diagnostic cytology. $7^{\text {th }}$ ed. Chicago:Tutorials of Cytology, 1992.

\begin{tabular}{l|l} 
& \\
& Endereço para correspondência \\
\hline & Lúcia de Noronha \\
& Laboratório de Patologia Experimental \\
Centro de Ciências Biológicas e da Saúde & \\
Campus 1 \\
Pontifícia Universidade Católica do Paraná \\
Rua Imaculada Conceição 1.155 - Prado Velho \\
CEP 80215-901 - Curitiba-PR \\
Tel.: (41) 330-1515 ramal 2264 \\
Fax: (41) 330-1624
\end{tabular}

